\title{
The role of serum \\ haemagglutination-inhibiting antibody in protection against challenge infection with influenza $A 2$ and $B$ viruses
}

\author{
By D. HOBSON AND R. L. CURRY \\ Department of Medical Microbiology, University of Liverpool
}

A. S. BEARE

M.R.C. Common Cold Research Unit, Salisbury

AND A. WARD-GARDNER

Medical Department, Esso Refinery, Fawley

(Received 17 May 1972)

\section{SUMMARY}

The intranasal inoculation of volunteers with living partially attenuated strains of influenza A and B viruses offers a new opportunity to determine the protective effect of serum haemagglutin-inhibiting antibody against a strictly homologous virus, under conditions where the time and dosage of the infective challenge can be controlled, the scoring of proven infections can be more precise and higher rates of infection can be achieved than in most natural epidemics.

In 1032 adult volunteers, whose serum HI antibody titre was determined immediately before virus challenge, there was a consistent inverse quantitative relationship between the HI titre and the likelihood of infection. The PD50 (50\% protective dose) of $\mathrm{HI}$ antibody was $1 / 18-1 / 36$, but an unusual finding was that volunteers with no detectable pre-challenge antibody often seem to be less susceptible to infection than those with pre-challenge antibody in low titre.

In one group of volunteers challenged with an influenza $B$ strain there was no evidence that pre-challenge antibody titres against viral neuraminidase had any significant protective effect against challenge infection.

\section{INTRODUCTION}

Within recent years it has become increasingly apparent that infection or vaccination of man with influenza viruses can induce quite separate antibodies against two distinct virus proteins, the haemagglutinin (HA) and neuraminidase (Nase), and that antibodies of each specificity may be found both in the IgG class of immunoglobulins in the bloodstream and in the IgA class in nasal secretions. The frequency of induction, the titres and the duration of each type of antibody in each location can vary quite independently (Schild \& Newman, 1969; Downie, 1970). It is still not clear which of these antibodies or which combination of antibodies is essential for protection against natural infective risk in man. In recent small-scale volunteer studies it has been shown that nasal 
antibodies against the HA antigen (Downie \& Stuart-Harris, 1970) and serum antibody against the Nase antigen (Slepushkin et al. 1971) may play some part in protection against experimental challenge infection with influenza viruses, whereas for the past 20 years it has been customary to regard serum antibody against the $\mathrm{HA}$ antigen as the essential protective agent, and in fact all commercially available influenza vaccines are designed and assessed on their ability to produce high sustained titres of haemagglutinin-inhibiting (HI) antibody.

It can readily be shown in the laboratory that the HI titre of a serum corresponds closely with its neutralizing activity against the infectivity of the homologous virus for chick embryo or tissue cultures (Hoyle, 1968). Nevertheless, surprisingly conflicting data have been reported on the protective effect of circulating HI antibody in man (e.g. Bashe, Stegmuller, Leonida \& Greenwald, 1964; Hoyle, 1968; Maynard et al. 1968). Many of the discrepancies are undoubtedly due to the inherent difficulty of studying small groups of people exposed to natural infective risk. The time of onset of an epidemic after the HI titres of a population have been recorded is unpredictable; the epidemic strain of virus may show antigenic drift from the strain used for HI titrations; rates of infection vary from epidemic to epidemic, and are often below $5 \%$; the exact virus challenge dose cannot be calculated, but must certainly be extremely variable from person to person.

Since serum HI antibody titrations are by far the simplest and still the most widely used method for epidemiological surveys and for estimating the efficacy of influenza vaccines in man, it seemed worth while to re-examine the relationship between HI titres and protection against influenza in a large number of adult volunteers, under conditions where the exact timing and dosage of infection by a strictly homologous virus strain could be carefully controlled, and where the total score of infections could be expected to be much higher and more easily proven than under field conditions in natural outbreaks.

In various clinical trials under the auspices of the Medical Research Council over 1000 volunteers have been given a deliberate intranasal challenge with known laboratory strains of influenza $A$ and $B$ virus. The rate of infection has been assessed by increases in the titre of homologous serum HI antibody and also, in several trials, by virus excretion studies after the challenge virus inoculation and by questionnaires about clinical signs and symptoms. The immediate purpose of these trials was to compare the infectivity of various virus strains, or to assess the efficiency of various influenza virus vaccines.

It now seems that a more detailed survey of previously unanalysed data from the whole series of trials would provide a unique opportunity to examine on a numerically larger scale than hitherto whether serum HI antibodies can still be regarded as a useful measure of the protection of a community against influenza, and would provide useful background data against which to evaluate recent claims that protection against influenza depends upon other antibodies of different specificity or distribution. 


\title{
Clinical trial procedures
}

\author{
MATERIALS AND METHODS
}

The present data are derived from two types of trial: $(a)$ challenge experiments in small groups of segregated volunteers in the Common Cold Unit, Salisbury, of the pattern previously described by Tyrrell (1963) and Beare et al. (1971); (b) larger-scale field trials in industrial workers who were divided randomly into groups some of which received various live or killed influenza vaccines, whilst others were given placebo materials or were left unvaccinated (see Beare et al. $1968,1969)$. The challenge infections analysed in the present paper were instituted 2-3 weeks after any schedule of vaccination was completed. Serum samples were taken before and 2 weeks after inoculation of the challenge virus, and nasal swabs were taken for virus isolation studies wherever possible $48 \mathrm{hr}$. after challenge.

The challenge infection was with chick embryo-grown pools of either B/Eng/13/65, or with the pre-Hong Kong A2 influenza virus strains A2/Leningrad/ $4 / 62$ or $A 2 / E n g / 501 / 68$, or with $A 2 / H o n g ~ K o n g / 1 / 68$ or strains related to it, e.g. A2/Eng/878/68. A small number of the Salisbury volunteers were inoculated with genetic recombinant strains of influenza A containing both the HA and Nase antigens of Hong Kong virus (Beare et al. 1971). The infective challenge dose was administered by nasal spray or droplets in doses of $10^{5}-10^{6}$ EID 50 per volunteer, as described previously (Beare et al. 1969). A positive challenge was scored in any volunteer who showed a fourfold or greater rise in serum HI titre against the homologous virus, or from whom virus was isolated in chick embryos or in monkey kidney tissue culture.

\section{Serological procedures}

HI antibody was titrated in cholera filtrate-treated sera by the World Health Organization (1953) technique, with $4 \mathrm{HA}$ units of virus incubated $1 \mathrm{hr}$. at room temperature with serum dilutions before adding $0.5 \%$ fowl erythrocytes.

Serum antineuraminidase (NI) antibody was investigated in only one group of volunteers challenged with $\mathrm{B} / \mathrm{Eng} / \mathbf{1 3} / 65$. The Nase assay was the modification of Warren's method described by Webster \& Laver (1967) using fetuin as the substrate. Assays of NI antibody were similar to those described by Schild \& Newman (1969) but with the following modifications; (a) the time of interaction of Nase and fetuin was $18 \mathrm{hr}$. at $37^{\circ} \mathrm{C}$; $(b)$ viral Nase and serum were incubated for $1 \mathrm{hr}$. at $37^{\circ} \mathrm{C}$. during enzyme inhibition; (c) the source of Nase was B/Lee which had been treated by $0.5 \%$ sodium dodecyl sulphate (Laver, 1963) to disrupt the virion and separate the HA and Nase components. This procedure was necessary to ensure that HI antibody would not produce non-specific inhibition of the enzyme by steric hindrance, as may happen on the intact virus particle (Easterday, Laver, Pereira \& Schild, 1969). Titres of NI antibody were expressed as that dilution of the volunteer's serum which reduced the activity of neuraminidase to $50 \%$ of its value in controls with normal rabbit serum. 


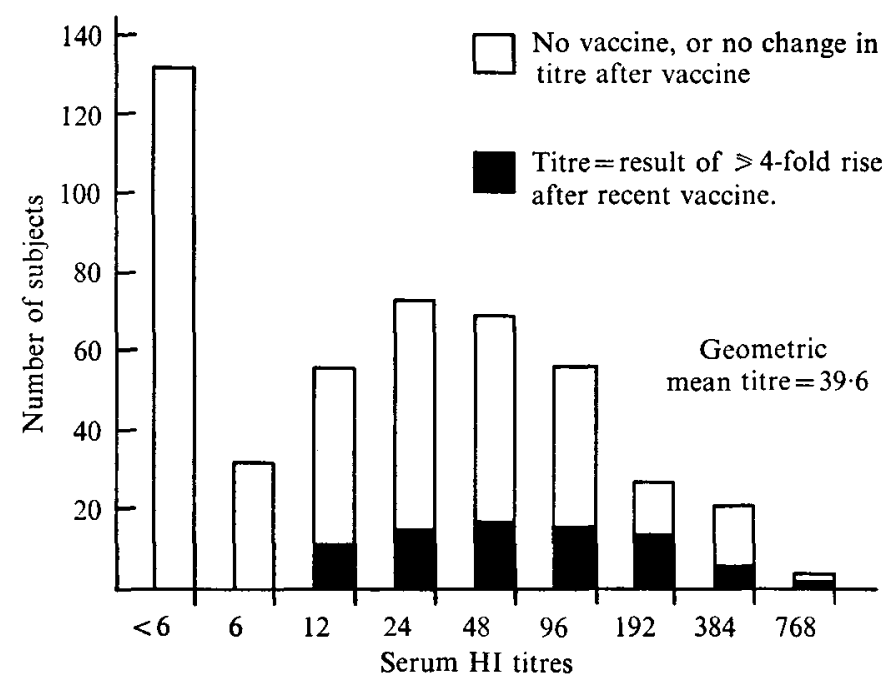

Fig. 1. The distribution of serum $H I$ antibody titres to $B / E n g / 13 / 65$ virus in 462 volunteers before homologous challenge infection.

\section{RESULTS}

\section{Influenza B trials}

The results of trials in three large industrial groups were closely similar and have been combined. The distribution of homologous HI serum antibody titres in 462 volunteers immediately before challenge infection are shown in Fig. 1. About $29 \%$ of the volunteers were without detectable HI antibody and the rest showed a Gaussian distribution of titres, with a geometric mean value of 1/39.6. Most of the volunteers had not previously been given vaccine, and their titres presumably represent residual antibody from natural infection in earlier years. However, as shown in Fig. 1 , in about $17 \%$ of volunteers the antibody titre at the time of challenge was the result of a fourfold or greater increase due to recent vaccination. This vaccine-enhanced group will later be discussed separately, because of the possibility that newly induced $\mathrm{HI}$ antibody might be of a different class or avidity from 'old' antibody, and because a recent antigenic stimulus might have resulted in antibodies, additional to serum HI antibody, which might not have persisted in the main group with 'old' HI antibody.

The number of infections in persons with no or low titres of antibody was greater than in those with high HI titres (Fig. 2), but it was difficult to see whether there was any consistent trend, because of the varying totals of people at each particular titre. When the results were recalculated (Fig. 3) in terms of the infection rate per cent of volunteers at each pre-vaccination antibody titre it was apparent that the likelihood of infection diminished progressively with increasing antibody titre. The PD 50 of HI antibody (i.e. the titre at which the infection rate is reduced to half the maximum observed rate) was approximately $1 / 18$, though it may be noted for later discussion that the infection rate in those with no detectable antibody was in fact very little higher than in those with the lowest detectable titre (1/6). When the group of people with 'old' antibody were considered separately from 


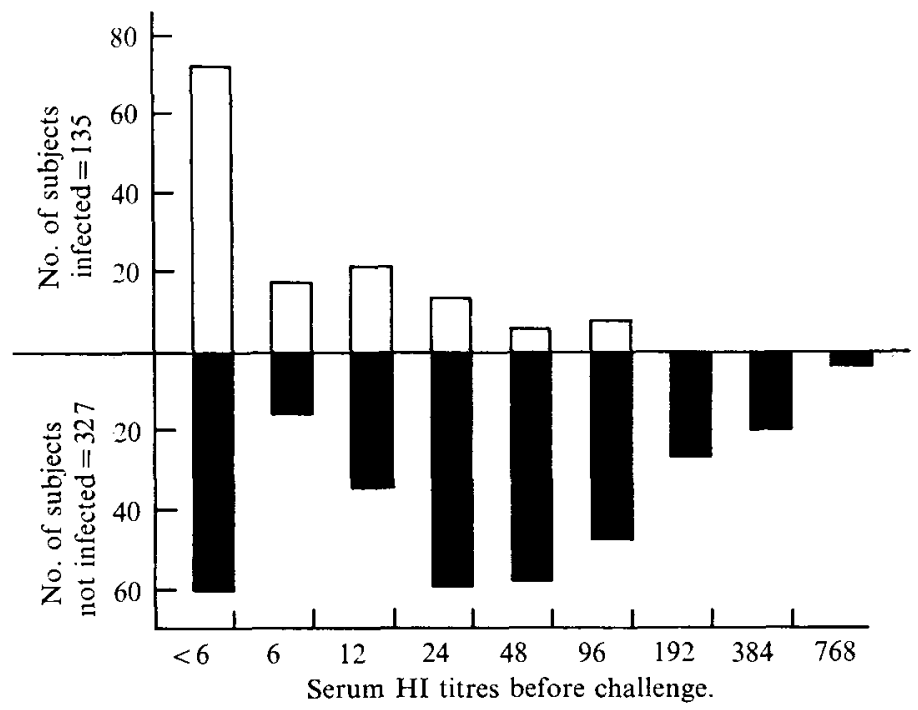

Fig. 2. The incidence of infection after challenge with $B / E n g / 13 / 65$ virus in volunteers with differing pre-challenge titres of serum HI antibody

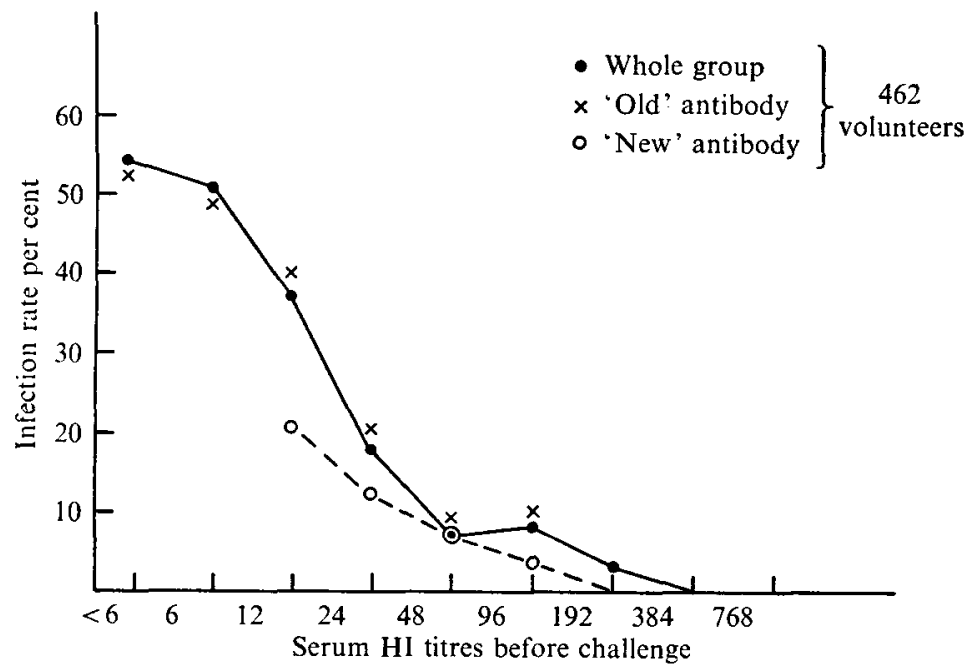

Fig. 3. The infection rates of volunteers challenged with $B / E n g / 13 / 65$ virus, in relation to their pre-challenge titres of serum $\mathrm{HI}$ antibody.

those with 'new' antibody it appeared that both types of antibody, titre for titre, indicate similar degrees of protection against challenge.

The results suggest that serum $\mathrm{HI}$ antibody confers protection, or alternatively that it is a good indirect quantitative indicator of some other factor such as NI antibody which is itself protective.

In one group of industrial volunteers the distribution of antibody against the neuraminidase of influenza type B viruses was compared with that of HI antibody to B/Eng/13/65. There was little or no correlation between HI and NI titres in individual sera, and many of those without detectable $\mathrm{HI}$ antibody against 


\begin{tabular}{|c|c|c|c|c|c|}
\hline \multicolumn{3}{|c|}{ HI titres } & \multicolumn{3}{|c|}{ NI titres } \\
\hline 384 & & & - & • & 320 \\
\hline 192 & & & & & 280 \\
\hline 96 & & $\bullet$ & - & $\bullet$ & 240 \\
\hline 48 & & $\bullet \bullet \bullet$ & & & 200 \\
\hline 12 & • & $\bullet \bullet$ & & $\bullet$ & \\
\hline 6 & & $\bullet$ & & & 80 \\
\hline$<6$ & $\bullet \bullet \bullet \bullet$ & & & -・ & 40 \\
\hline \multirow[t]{2}{*}{$\begin{array}{l}\text { Geometric } \\
\text { mean titre }\end{array}$} & $<6$ & 49 & 91 & 96 & \\
\hline & Infected & Not infected & Infected & Not infected & \\
\hline
\end{tabular}

Fig. 4. The distribution of $\mathrm{HI}$ and NI serum antibodies in 29 volunteers before challenge infection with $\mathrm{B} / \mathrm{Eng} / 13 / 65$ virus.

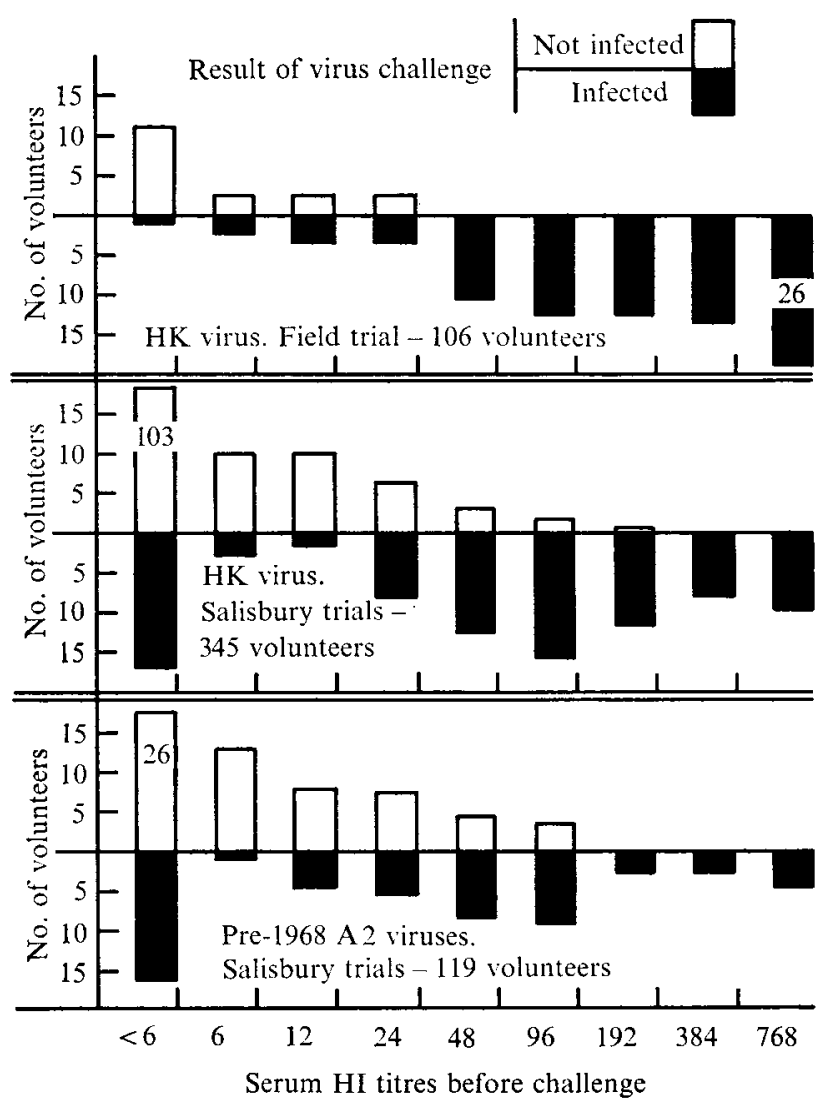

Fig. 5. The effect of challenge infection with A2 viruses in volunteers with differing pre-challenge titres of homologous serum $\mathrm{HI}$ antibody. 

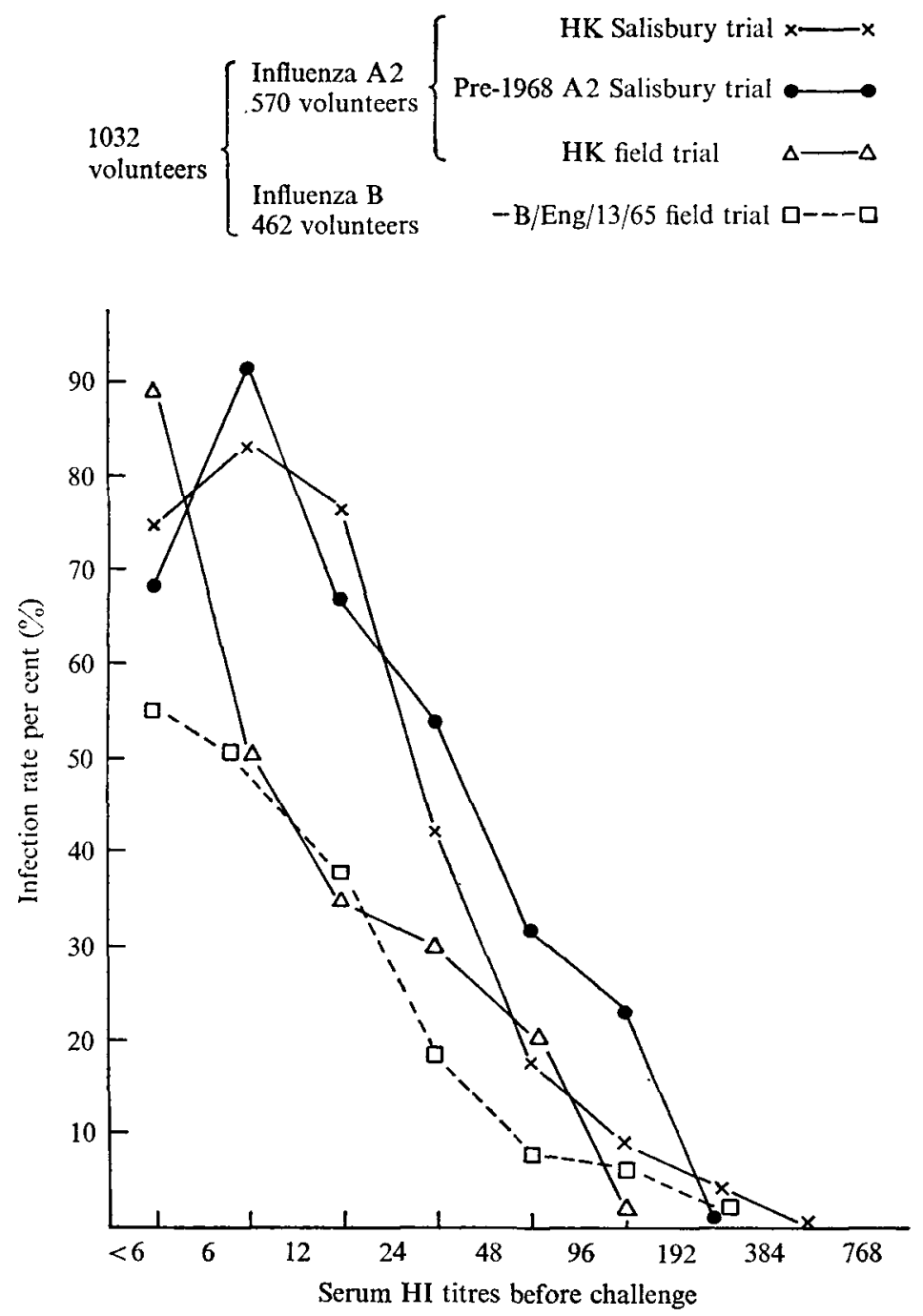

Fig. 6. The infection rate of volunteers challenged with various influenza viruses, in relation to their pre-challenge titres of homologous serum HI antibody.

B/Eng/13/65 had substantial NI activity against the type-common enzyme. It is probable that repeated previous natural infection of these adult volunteers with a variety of foregoing type B viruses had maintained antibody to the common enzyme, without inducing $\mathrm{HI}$ antibody to the distinctive HA antigen of the more recent B/Eng/13/65. The serum NI titre was not apparently correlated with protection against challenge infection in this small group of volunteers (Fig. 4), whereas HI titres were lower in those who became infected after challenge than in those who did not.

\section{Influenza $A$ trials}

A total of 570 volunteers were studied, comprising two industrial populations challenged with A2/Hong Kong/1/68 and Salisbury volunteers challenged with either pre-Hong Kong or Hong Kong-like strains of influenza A2 virus. The dis- 
tribution of homologous HI titres immediately before challenge is shown in Fig. 5, and the infection rate per cent of volunteers in terms of their initial antibody level is shown separately for each class of trial in Fig. 6. Only one group of industrial volunteers had previously received influenza vaccines, and the small number with a significant induction of fresh antibody before challenge is too small to merit separate discussion.

An unexpected anomalous finding in two of the three groups shown in Fig. 6 was that the rate of infection in those with no detectable antibody was noticeably less than in those with antibody at the lowest detectable level (1/6). However, in those subjects with antibody before challenge there was a progressive fall in infection rate with increasing serum $H I$ titres, as seen earlier in the influenza $B$ trials. The PD50 of serum HI antibody again appeared to be low, of the order of 1/18-1/36, both with the Hong Kong-like viruses against which antibodies could only have been induced in the preceding twelve months or so, and with the earlier A2 strains with which the community had had repeated immunogenic experiences over several years.

\section{DISCUSSION}

The results of challenge infection of 1032 adult volunteers with influenza A2 or $B$ viruses show that the distribution of homologous $H I$ antibodies in the community immediately before challenge reflects the distribution of subsequent infections closely and consistently. However, observational data of this type can obviously not confirm that a quantitative relationship between antibody and resistance to infection is a causal relationship. In many cases the $\mathrm{HI}$ antibody is only part of a wider immune response to each of the distinct antigens of the influenza virus, and in the short term serum NI antibody and nasal NI and HI antibodies may persist alongside the more readily measured serum HI antibody. The apparent lack of correlation between HI and NI antibodies in the sera of one of the present groups is of interest, and the evidence suggests that the former is more closely correlated with infection than the latter. It is possible that NI antibody is of less importance in protection against influenza $B$ virus infections than against influenza $A$ as has been suggested by Slepushkin et al. (1971), but a much larger scale survey than the present study would be required to evaluate the inter-relationship of the two antibodies in protection against influenza.

The low titre of serum HI antibody which appears to give significant protection against infection (i.e. the PD 50 titre) may suggest that other protective factors are also involved, but it is possible that the low PD 50 merely reflects the essential requirement of the present trials that the challenge virus strains should be selected chick embryo-grown variants of somewhat attenuated virulence, likely to produce less persistent or clinically severe infections than parental wild-type strains. It is thus not possible to extrapolate from the present experimental procedures to forecast that similar low titres of serum HI antibody would protect against natural infection with highly virulent epidemic strains of virus. However, it is of interest that Meiklejohn, Kempe, Thaiman \& Lennette (1952) calculated that in a natural influenza A1 epidemic in servicemen the percentage attack rate 
in those with pre-epidemic homologous $\mathrm{HI}$ antibody titres of $1 / 16$ was only $1.5 \%$ as compared with $18 \%$ in those without detectable antibody.

The low PD 50 of antibody shown in the present trial suggests that the development of vaccines capable of inducing very high HI titres of 1/1000 or greater, which has been the aim of manufacturers in the past, may not be necessary as far as short-term protection is concerned, and may only be required, if at all, to ensure that the decline to subeffective titres after vaccination is postponed as long as possible. However, it is possible that naturally acquired serum HI antibody, as in the majority of the present volunteers, may be quantitatively different from that induced by vaccine or that the ratio of NI antibody and nasal antibodies to serum HI antibody is greater after natural infection than after vaccination.

An anomalous finding in the present study was the apparently lower susceptibility to challenge infection in those without serum $\mathrm{HI}$ antibody than in those with antibody at the minimum detectable level of $1 / 6$. Further larger-scale investigations would be necessary to show whether this is merely a chance finding due to small numbers, or is peculiar to these particular attenuated virus strains. However, similar discrepancies have been found in field trials with live influenza vaccines (McDonald, Zuckermann, Beare \& Tyrrell, 1962). A simple explanation could be that those without detectable antibody are a heterogeneous group comprising those who have never been infected and thus have no relevant clone of committed lymphocytes, together with those who have been immunized previously, but in whom the rate of production of specific immunoglobulins has fallen to levels undetectable in the HI test. In this case, it might be expected that the latter subgroup would respond to infection like those already showing antibody, i.e. by a rapid immunological recall and production of readily detectable titres of new antibody, whereas those in the truly unsensitized subgroup who become infected would show only a primary immune response, of insufficient size to be detected in the present test. There would thus be a difference in the precision of scoring the infection rates in the two subgroups. An earlier trial (Hobson, Lane, Beare \& Chivers, 1964) with adjuvant inactivated influenza vaccine showed that the immunological response, in terms of the HI test, of a group of people who were all devoid of pre-vaccine HI antibody was greater in those with previous sensitization, detectable by a more sensitive tissue culture neutralization test, than in those who were absolutely inexperienced.

However, the limited virus isolation studies in the present trials suggest that antibody-negative volunteers show not merely a deficient response to infection, but a reduced rate of infection. Thus, the intriguing possibility exists that the fraction of the population which is found to be apparently unimmunized several years after a particular subtype of influenza has become prevalent in the community may be a naturally selected 'resistant' group rather than a random collection of people who have merely escaped influenza by chance.

It is well known that resistance to other human infective diseases, e.g. tuberculosis, may be determined by many genotypic and phenotypic factors, and in influenza itself it has been suggested (McDonald \& Zuckerman, 1962; Potter, 1969) that the susceptibility of people with Blood Group A is lower than those of 
Group $\mathrm{O}$ constitution. Similarly, there are many instances of genetically controlled differences of mice to many microbial infections (Allison, 1965), including influenza (Lindenmann, Lane \& Hobson, 1963).

It would be of interest in future epidemiological surveys to determine whether those who continue to escape influenza in natural epidemics, despite an apparent lack of serum HI antibody, are different from the general population in certain specific ways. It is possible that this group may be incapable of making serum HI antibody whilst remaining immunologically competent to make antibody to viral neuraminidase, but it is equally possible that their relative resistance to infection may be due to non-immunological factors, such as a greater facility for interferon production or inhibitor-secretory activity, or because they may develop some form of cell-mediated immunity which may be more closely correlated with resistance than is their serological status.

It is a pleasure to express our gratitude to Professor Sir Charles Stuart-Harris, Dr D. A. J. Tyrrell and other members of the Influenza and other Respiratory Virus Vaccines Committee of the Medical Research Council for facilitating these studies and for their advice on their presentation.

\section{REFERENCES}

Altrson, A. C. (1965). Genetic factors in resistance against virus infections. Archiv für die gesamte Virusforschung 17, 280.

Bashe, W. J., Stegmuller, H., Leonida, D. \& Greenwatd, P. (1964). Failure of polyvalent vaccine to provide clinical protection against Asian influenza. New England Journal of Medicine 270, 870 .

Beare, A. S., Hobson, D., Reed, S. E. \& Tyrrell, D. A. J. (1968). A comparison of live and killed influenza virus vaccines. Lancet ii, 418.

Beare, A. S., MaAssab, H. F., Tyrrell, D. A. J., Slepushkin, A. N. \& Hall, T. S. (1971). A comparative study of attenuated influenza viruses. Bulletin of the World Health Organisation 44, 593.

Beare, A. S., Trrrell, D. A. J., Hobson, D., Howells, C. H. L., Perema, M. S., Pollock, T. M. \& Tyler, L. E. (1969). Live influenza B vaccine in volunteers. Journal of Hygiene 67, 1.

DowNIE, J. C. (1970). Neuraminidase-inhibiting and haemagglutination-inhibiting antibodies in serum and nasal secretion of volunteers immunized with attenuated and inactivated influenza B/Eng/13/65 virus vaccines. Journal of Immunology 105, 620 .

Downie, J. C. \& STUART-HarRIS, C. H. (1970). The production of neutralizing activity in serum and nasal secretion following immunization with influenza B virus. Journal of Hygiene 68, 233.

Easterday, B., Laver, W. G., Pereira, H. G. \& Schild, G. C. (1969). Antigenic composition of recombinant virus strains produced from human and avian influenza viruses. Journal of General Virology 5, 83.

Hobson, D., Lane, C. A., Beare, A. S. \& Chivers, C. P. (1964). Serological studies on adult volunteers inoculated with oil-adjuvant Asian influenza vaccine. British Medical Journal ii, 271.

Hoyцe, L. (1968). The Influenza Viruses, chap. 27. Vienna: Springer-Verlag.

Laver, W. G. (1963). The structure of influenza viruses. 3. Disruption of the virus particle and separation of neuraminidase activity. Virology 20, 251.

Lindenmann, J., Lane, C. A. \& Hobson, D. (1963). The resistance of A2G mice to myxoviruses. Journal of Immunology $90,942$.

McDonald, J. C., Zuckermann, A. J., Beare, A. S. \& Tyrrell, D. A. J. (1962). Trials of live influenza vaccine in the Royal Air Force. British Medical Journal i, 1036. 
McDonald, J. C. \& Zuckermane, A. J. (1962). A B O blood groups and acute respiratory virus disease. British Medical Journal ii, 89.

Maynard, J. E., Duld, H. B., Hanson, M. L., Feltz, E. T., Berger, B. \& Hammes, L. (1968). Evaluation of monovalent and polyvalent influenza vaccines during an epidemic of Type A2 and B influenza. American Journal of Epidemiology 87, 148.

Meiklejohn, G., Kempe, C. H., Thalman, W. G. \& LennetTe, E. H. (1952). Evaluation of monovalent influenza vaccines. II. Observations during an influenza A-prime epidemic. American Journal of Hygiene 55, 12.

PotTer, C. W. (1969). HI antibody to various influenza viruses and adenoviruses in individuals of blood groups A and O. Journal of Hygiene 67, 67.

Schild, G. C. \& Newman, R. W. (1969). Antibody against influenza A2 virus neuraminidase in human sera. Journal of Hygiene 67, 353.

Slepushkin, A. N., Schild, G. C., Beare, A. S., Chinn, S. \& Tyrreli, D. A. J. (1971). Anti-neuraminidase antibody and resistance to vaccination with live A2/Hong Kong influenza virus vaccines. Journal of Hygiene 69, 571.

Tyrrell, D. A. J. (1963). The use of volunteers. American Review of Respiratory Diseases 88, Supplement 128.

Webster, R. G. \& LAver, W. G. (1967). Preparation and properties of antibody directed specifically against the neuraminidase of influenza virus. Journal of Immunology 99, 49.

World Health Organization (1953). Expert Committee on Influenza. Technical Report Series of the World Health Organization, no. 64. 